\title{
EDIFICATE
}

I Congreso de Escuelas de Edificación y Arquitectura Técnica de España València, 4 y 5 de noviembre de 2021

Escuela Técnica Superior de Ingeniería de Edificación

Universitat Politècnica de València

Doi: https://doi.org/10.4995/EDIFICATE2021.2021.13251

\section{La Inteligencia Artificial y su contribución a la optimización de la atención y memoria de los estudiantes a través del diseño del aula}

\author{
Artificial Intelligence and its contribution to optimising \\ students' attention and memory through classroom design.
}

\begin{abstract}
Adrián Colomer Granero ${ }^{a}$, Juan Luis Higuera Trujillo ${ }^{b}$, Valeriana Naranjo Ornedoc y Carmen Llinares Millán ${ }^{d}$

anstituto de Investigación e Innovación en Bioingeniería, adcogra@i3b.upv.es, 'Escuela Técnica Superior de Ingeniería de la Edificación, jlhiguera@i3b.upv.es, 'Instituto de Investigación e Innovación en Bioingeniería, vnaranjo@dcom.upv.es y ${ }^{\mathrm{E} E s c u e l a ~ T e ́ c n i c a ~ S u p e r i o r ~ d e ~ I n g e n i e r i ́ a ~ d e ~ l a ~ E d i f i c a c i o ́ n, ~}$ cllinare@omp.upv.es
\end{abstract}

\begin{abstract}
The application of Artificial Intelligence to education has been studied for decades. However, despite its numerous contributions, there are hardly any studies focused on improving student performance through classroom design. The aim of this paper is to apply Artificial Intelligence to identify the optimal classroom design (based on colour, lighting and shape) to enhance the attention and memory performance of university students. To this end, a laboratory study was carried out in which 50 participants performed cognitive tests in virtual environments with different design configurations. The results show that the best performance in attention and memory is achieved with a similar design: smaller classrooms (ceiling height of $2.6 \mathrm{~m}$ and width of $7.6 \mathrm{~m}$ ), with cool colours and low saturations, and with lighting colour temperatures of $6500 \mathrm{~K}$. The only difference is in the illuminance: it requires lower levels (1001x) to enhance memory; and higher levels (500lx) for attention. The results may be useful for both researchers and professionals involved in the design of educational centres.
\end{abstract}

Keywords: classroom design, attention, memory, artificial Intelligence. 

and memory through classroom design.

\section{Resumen}

La aplicación de la Inteligencia Artificial en el sector de la educación ha sido objeto de estudio durante décadas. Sin embargo, y a pesar de sus numerosas contribuciones, apenas existen estudios centrados en mejorar el rendimiento de los estudiantes a través del diseño del aula. El objeto del presente trabajo es la aplicación de la Inteligencia Artificial para identificar el diseño óptimo del aula (atendiendo a su color, iluminación y forma), para potenciar el rendimiento en atención y memoria de los estudiantes universitarios. Para ello se llevó a cabo un estudio en laboratorio en el que 50 participantes realizaron pruebas cognitivas en entornos virtuales con diferentes configuraciones de diseño. Los resultados muestran que el mejor rendimiento en atención y memoria se consigue con un diseño similar: aulas de menores dimensiones (altura de techo de 2,6 $m$ y 7,6 $m$ de ancho), con colores fríos y de saturaciones bajas, y con temperaturas del color de la iluminación de 6500K. La única diferencia se detecta en la iluminancia: requiere niveles más bajos (100lx) para potenciar la memoria; y más altos (500lx) para la atención. Los resultados pueden ser de utilidad tanto para investigadores como profesionales relacionados con el diseño de centros docentes.

Palabras clave: diseño aulas, atención, memoria, inteligencia artificial 


\section{Introducción}

El término de Inteligencia Artificial no existía como tal hasta que en 1956 fue utilizado por primera vez en el congreso de Dartmounth, organizado por diversos investigadores interesados en desarrollar máquinas que imitaran el comportamiento humano. En dicha conferencia, el Profesor John McCarhty de la Universidad de Stanford utilizó el nombre de Inteligencia Artificial para referirse a "la ciencia y el ingenio de hacer máquinas inteligentes, especialmente programas de ordenador inteligentes".

Tras varios experimentos fallidos en torno a la Inteligencia Artificial a lo largo de las décadas de los 70 y 80 , se extrajo una conclusión clave: antes de crear ordenadores inteligentes, era necesario saber cómo proporcionarles la información requerida. Dado que este conocimiento no podía ser proporcionado enteramente por los seres humanos, las máquinas tenían que aprender por ellas mismas a partir del entorno, ya fuera utilizando bases de datos o motores de búsqueda. Capturar información del entorno es de vital importancia para la inteligencia artificial. Hoy en día, se genera diariamente una gran cantidad de información en todos los ámbitos (incluido el sector de la educación), haciendo del Big Data una técnica esencial en el siglo XXI (Henke, 2021; Reinsel et al., 2018).

En las últimas décadas, gracias al avance de la tecnología hardware se han creado sistemas revolucionarios de Inteligencia Artificial en diversos ámbitos. Muchas áreas de la vida cotidiana, tales como el sector educación, automovilístico, industrial, médico, aeronáutico y financiero entre otros piden asistentes digitales que permitan reducir costes. Esta demanda ha forzado la creación de los que se conoce como Inteligencia Artificial 2.0. En esta dirección, se proponen dos objetivos principales. El primero consiste en alcanzar sistemas inteligentes híbridos que combinen máquinas y humanos; el segundo supone crear sistemas inteligentes más complejos, conocidos comúnmente como sistemas supervisados (Pan, 2016).

La aplicación de la Inteligencia Artificial en el sector de la educación (también conocida como AIED, de sus siglas en inglés) ha sido objeto de estudio durante décadas (Rus et al., 2013). Uno de los objetivos principales es el de proporcionar una comprensión más profunda de como el aprendizaje del alumnado tiene lugar y de la influencia que en el ejercen otros factores tales como el contexto físico y socioeconómico de la persona.

Los Sistemas de Tutorización Inteligente (STI) tienen una serie de conocimientos, tanto de los contenidos y de los estudiantes como de las metodologías de aprendizaje, que les permiten ayudar al estudiante guiándolo a través del proceso de aprendizaje y la resolución de problemas. Los STI utilizan técnicas de IA para simular la tutorización individualizada, ofreciendo las actividades que mejor encajan con las necesidades de cada alumno y proporcionando retroalimentación (Roll et al., 2016). Algunos STI buscan motivar a los estudiantes a tomar el control de su propio aprendizaje, esperando desarrollar su responsabilidad y madurez. Los STI existentes hoy en día usan técnicas de aprendizaje automático, algoritmos de autoaprendizaje y redes neuronales para tomar las decisiones apropiadas sobre qué contenido proporcionar al alumno (Vanlehn, 2011). 
Otra alternativa a estos tutores inteligentes son los sistemas de evaluación automáticos (Lama et al. 2007), los cuales además de corregir al estudiante, le permiten conocer sus fortalezas y debilidades. Entre las alternativas a este modelo de aprendizaje están los ToL, basados en test online (Tartaglia et al. 2002), y CELLA, para el estudio del inglés (Lama et al. 2007).

Aparte de los sistemas mencionados previamente, hay otros que permiten aprender no en término de materias sino en términos de aptitudes. Éste es el caso del aprendizaje colaborativo, que enseña y guía a los estudiantes para desarrollar su capacidad de trabajo en grupo y mejorar la interacción entre ellos, como es el caso de DEGREE (Barros et al. 2000). Otra forma más dinámica de aprendizaje es aquella basada en los juegos (Lama et al. 2007). Con ellos, los estudiantes se ven motivados a desarrollar habilidades y conocimientos, e incluso les hacen estar más concienciados con problemáticas relacionadas con la sociedad y la ciudadanía, como en el caso del método de aprendizaje NetAid, cuyo objetivo es sensibilizar sobre la pobreza (Stokes, 2005).

Asimismo, parte de estos sistemas puede usarse tanto dentro de las aulas como fuera a través de Internet, permitiendo una educación continuada en cualquier momento y lugar.

Otra técnica implementada para apoyar el aprendizaje consiste en el uso de la realidad virtual, que puede proveer de experiencias inmersivas que simulan ciertos aspectos de la vida cotidiana. Esta función añadida a la Inteligencia Artificial permite al usuario interactuar y tomar decisiones en situaciones que podrían ser reales, registrando diferentes comportamientos. Dentro de estas experiencias, pueden asumir distintos roles. Por ejemplo, FearNot es un sistema de realidad virtual diseñado por escuelas y que presenta entornos relacionados con el acoso escolar. Este sistema busca ayudar a las personas que lo han sufrido dándoles consejos sobre que comportamientos adoptar en distintas situaciones (Vannini, 2011).

La AIED utilizada en las aulas ha evolucionado, y actualmente se persigue el objetivo de diseñar e implementar aulas inteligentes ("Smart classrooms en inglés"). Es posible combinar sistemas cognitivos con técnicas de minado de datos para hacer un seguimiento del comportamiento de los estudiantes y de su nivel de atención y concentración en clase, por ejemplo, a través de la recolección de información sobre su asistencia y publicando estudios para identificar y dar soporte a los estudiantes en riesgo de abandono escolar (Timmins, 2016). Otros investigadores están trabajando en nuevas interfaces, tales como el procesamiento del lenguaje natural, habla, reconocimiento de gestos y eye-tracking, entre otras, que podrían aplicarse al campo de la educación.

Sin embargo y a pesar de las numerosas aplicaciones que ha tenido esta técnica apenas existen estudios cuyo objetivo haya sido identificar elementos de diseño de un espacio para la mejora del rendimiento de los estudiantes. La contribución radica fundamentalmente en la posibilidad de identificar la combinación de diferentes elementos de diseño, y no el efecto de cada elemento de manera aislada o independiente como se ha realizado hasta el momento. 


\section{Objetivo}

El objeto del presente trabajo es la aplicación de la Inteligencia Artificial para identificar el diseño óptimo del aula (atendiendo a su color, iluminación y forma), capaz de potenciar las funciones cognitivas de atención y memoria de los estudiantes universitarios. Entre el conjunto de parámetros de diseño con capacidad de influir en las funciones cognitivas de los estudiantes en este trabajo nos centramos en el color (tono y saturación), forma (altura techo y anchura aula) e iluminación (iluminancia y temperatura del color).

\section{Material y Métodos}

La metodología se basó en un estudio en laboratorio, con las siguientes características.

\subsection{Muestra}

La muestra estaba formada por 50 participantes (55\% hombres- $45 \%$ mujeres), con una edad media de 20.06 años $(\sigma=3.122)$. Para formar parte de la muestra se marcaron cuatro criterios de inclusión: ser estudiante universitario, español (para evitar posibles efectos culturales), tener entre 18 y 23 años (edades más frecuentes en los grados universitarios de España) y no tener dificultades en la apreciación de los colores.

\subsection{Estímulos}

Como estímulo base se tomó la réplica virtual inmersiva de un aula física representativa. Concretamente, un aula de la Escuela Técnica Superior de Ingeniería de la Edificación (ETSIE) de la Universitat Politècnica de València. Sobre esta aula base se desarrollaron 34 configuraciones aisladas de diseño, atendiendo a las siguientes características:

- Variable forma. Con tres medidas para la altura del techo: $3.8 \mathrm{~m}, 3.2 \mathrm{~m}$ y $2.6 \mathrm{~m}$; y otras tres para la anchura: $8.8 \mathrm{~m}, 8.2 \mathrm{~m}$ y $7.6 \mathrm{~m}$.

- Variable iluminación. Con tres medidas para la iluminancia: $1001 x, 3001 x$ y 500lx; y otras tres para la temperatura del color: $3000 \mathrm{~K}, 4000 \mathrm{~K}$ y $6500 \mathrm{~K}$.

- Variable color. Con 8 tonos, divididos a su vez en fríos (5BG, 5PB, 5P, 5GY) y cálidos (5RP, 5R, 5YR, 5Y); y 2 saturaciones: alta (entre 10 y 16 unidades en Munsell chroma) y baja (entre 4 y 10 unidades).

Los participantes visualizaron las simulaciones mediante el dispositivo HTC Vive. Cada participante realizó 6 visualizaciones siguiendo una aleatorización automática; dando como resultado que cada estímulo fuese visto por al menos 8 participantes. La Figura 1 muestra una de las aulas evaluadas y una imagen de la experiencia. 

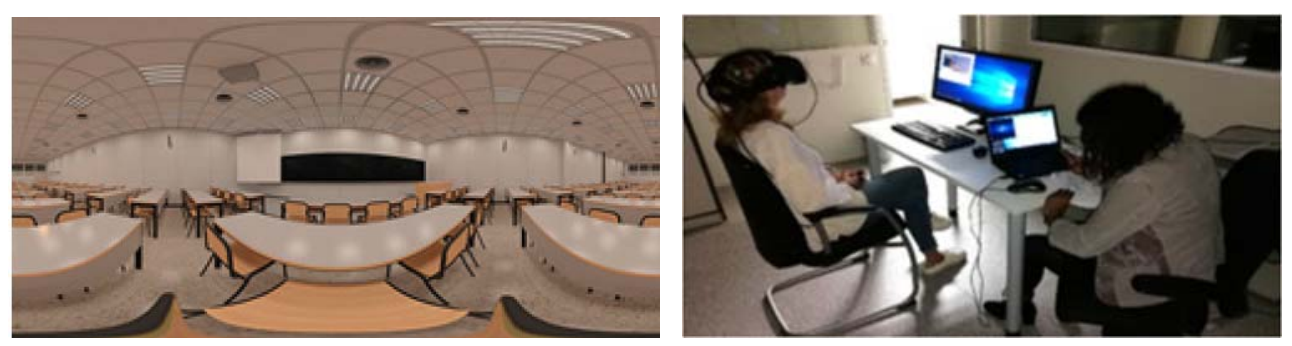

Fig. 1 Aula virtual y participante durante la experiencia

\subsection{Análisis de datos}

De cada participante se cuantificó el rendimiento en las tareas de atención y memoria.

Tarea de memoria. Esta tarea consistió en la memorización de un conjunto de palabras, basado en el estudio de Alonso y su equipo (2004). En concreto el participante debía escuchar 3 audios, cada uno de ellos con una lista de 15 palabras relacionadas entre sí. Las listas se presentaron contrabalanceadas. El participante debía escucharlas y repetirlas en un tiempo máximo de 30 segundos, antes de avanzar a la siguiente lista. Posteriormente se anotaba el número de aciertos de cada lista. El número de palabras recordadas fue corregido según la tasa de recuerdo propuesta por Alonso et al (2004).

Tarea de atención. Esta tarea consistía en reaccionar lo antes posible ante un estímulo auditivo concreto con un clic de ratón (objetivo) y evitar hacerlo ante otros cuatro (distractores), similar a las pruebas de rendimiento auditivo continuo (Seidman et al., 1998). En total la tarea contaba con 8 objetivos y 32 distractores, y el tiempo entre estímulos era de $800 \mathrm{~ms}$ a $1600 \mathrm{~ms}$. Los participantes tenían $750 \mathrm{~ms}$ para reaccionar a los estímulos, pasados los cuales se consideraba un error (al igual que reaccionar ante un estímulo distractor). Se registró el tiempo de reacción ante los estímulos objetivos y el número de errores cometidos. Esto se repetía en 3 ocasiones para cada entorno simulado, con 1500 ms entre sets.

\subsection{Inteligencia Artificial para identificar el diseño óptimo del aula}

Tal y como se ha comentado en la Sección 1, no existen estudios previos que focalicen en la identificación de elementos de diseño de un espacio para la mejora del rendimiento de los estudiantes mediante técnicas de Inteligencia Artificial. Para llevar a cabo el objetivo que se plantea en este trabajo, se siguió la metodología CRISP-DM para llevar a cabo proyectos de aprendizaje automático publicada por Shearer (2000), adaptándola al presente estudio (ver Figura 2)

En primer lugar, se realizó un proceso de comprensión de los datos adquiridos definido por varias tareas: descripción de los datos obtenidos, exploración de los mismos e identificación de su calidad. Tras esta primera etapa, se llevaron a cabo técnicas de selección y limpieza de datos para eliminar valores outliers, así como métodos de interpolación para imputar valores perdidos, garantizando así la construcción de una base de datos robusta y fiable. 
Posteriormente, y tomando como entrada la base de datos acondicionada, se dio comienzo a la fase del modelado del conocimiento a partir de dichos datos. Más concretamente, se emplearon técnicas de extracción manual de características y métodos estadísticos para el modelado. Tras esta etapa, se procedió a llevar a cabo una validación exhaustiva del modelo generado con un subconjunto de participantes cuyos datos no se emplearon en la etapa de construcción del modelo, garantizando así la habilidad de generalización y fiabilidad del mismo.

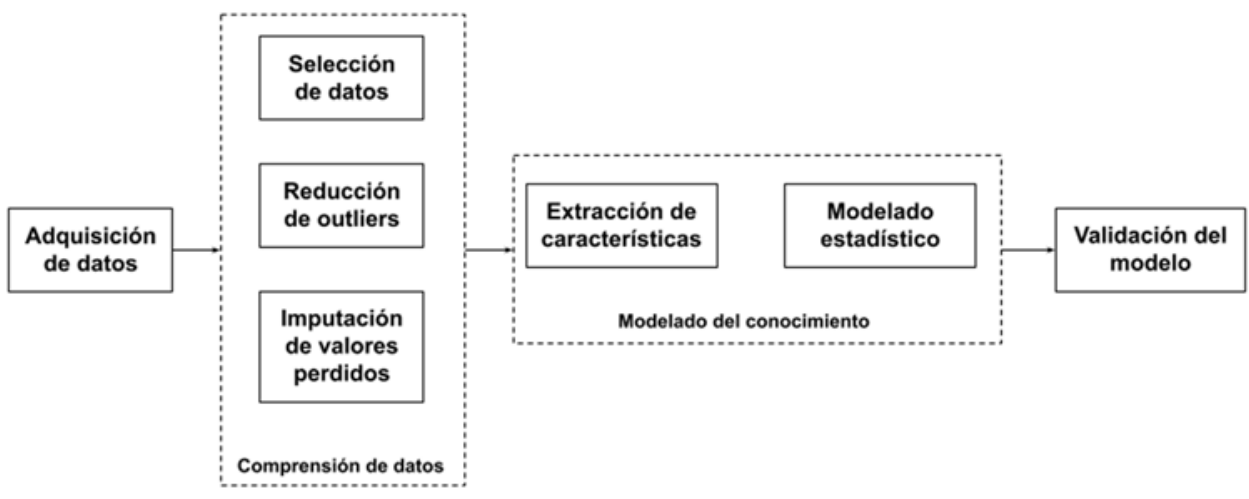

Fig. 2 Metodología CRISP-DM propuesta para la identificación de elementos de diseño de un espacio con el objetivo de mejorar el rendimiento de los estudiantes mediante técnicas de Inteligencia Artificial

\section{Resultados}

A partir de la aplicación de la Inteligencia Artificial se identificó la combinación de elementos de diseño que consigue maximizar las funciones cognitivas de atención y memoria. La Figura 3 recoge un esquema de los resultados.

Como se observa en el esquema siguiente el mejor rendimiento de la atención y de la memoria se consigue con un diseño similar: aulas de menores dimensiones (con una altura de techo de 2,6 $\mathrm{m}$ y 7,6 $\mathrm{m}$ de ancho), con colores fríos de saturaciones bajas, y con una temperatura del color de la iluminación de $6500 \mathrm{~K}$. La única diferencia se detecta en la iluminancia, que requiere niveles más bajos (100lx) para potenciar la memoria, y por el contrario el nivel más alto para potenciar la atención (500lx).

No existen trabajos previos que analicen la relación entre geometría del aula (altura-anchura) con respecto a las funciones cognitivas. Por el contrario, respecto al color existen trabajos previos como el de Mahnke (1996) que asocian los colores fríos a una mejora de la concentración de los estudiantes de más edad. También Stone (2003) y Xia et al. (2016) demostraron que los tonos azules mejoraban el desarrollo de tareas de gran dificultad. 
La Inteligencia Artificial y su contribución a la optimización de la atención y memoria de los estudiantes a través del diseño del aula- Artificial Intelligence and its contribution to optimising students' attention and memory through classroom design.

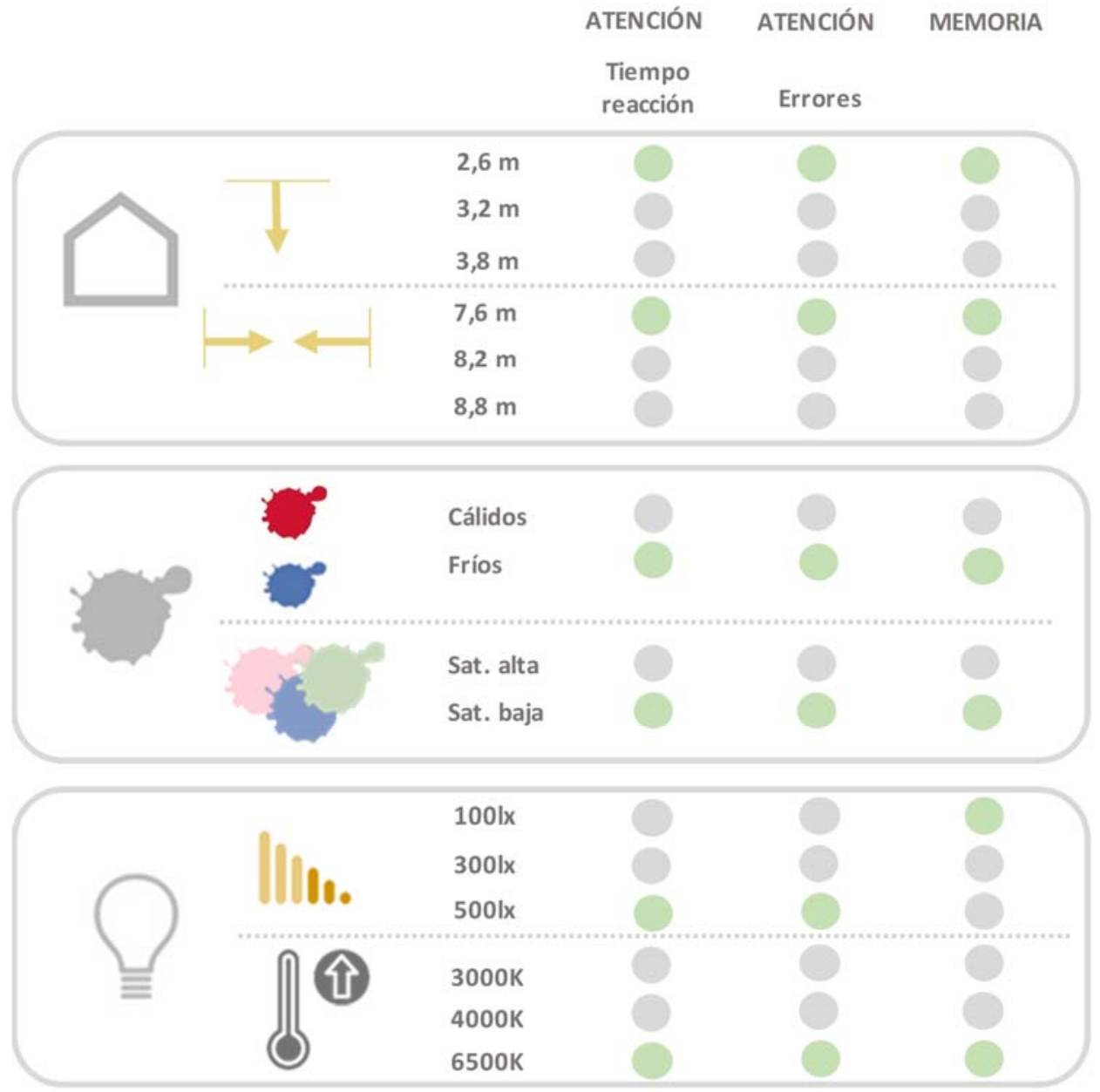

Fig. 3 Incidencia de la forma, el color y la iluminación en la atención y memoria de los estudiantes

La saturación del color ha sido también objeto de estudio. Así, por ejemplo, Kwallek et al. (1996) demostró que saturaciones similares a las etiquetadas como bajas en el presente estudio conseguían reducir errores en la ejecución de tareas intelectuales. En cuanto a la iluminación, son varios los trabajos que han observado una mejora de concentración con niveles elevados de temperatura del color (Viola et al., 2008; Keis et al., 2014). La discrepancia encontrada con la iluminancia es similar al resultado observado por Smolders y su equipo (2012), que ante un cambio del nivel de iluminación de 200lx a 1000lx mejoraron los niveles de atención sostenida y sin embargo, en algunas tareas de memoria el resultado era inverso, de forma que el mejor desempeño se producía con el nivel más bajo de iluminación (2001x). 


\section{Conclusiones}

La iluminación, el color y las dimensiones del aula son aspectos fundamentales a ser considerados en el diseño de los espacios arquitectónicos docentes. Son muchos los trabajos que han analizado la incidencia de un elemento de diseño, como la iluminación o el color, en el rendimiento de los estudiantes. Sin embargo, resulta fundamental analizar la incidencia de los elementos de diseño de manera combinada. Así por ejemplo la utilización de colores fríos en los revestimientos del aula potencia la atención y memoria del alumnado, pero siempre que éstos tengan saturaciones bajas. La Inteligencia Artificial permite realizar este análisis combinado. Los resultados pueden ser de utilidad tanto para investigadores como profesionales relacionados con el diseño de centros docentes.

\section{Agradecimientos}

Este trabajo ha sido financiado por el Ministerio de Economía, Industria y Competitividad de España (Proyecto BIA2017-86157-R; PRE2018-084051).

\section{Referencias}

ALONSO, M. Á., FERNANDEZ, Á., DIEZ, E., y BEATO, M. S. (2004). "Índices de producción de falso recuerdo y falso reconocimiento para 55 listas de palabras en castellano" en Psicothema, vol. $16, \mathrm{n}^{\circ}$ 3, pp. 357-362.

BARROS B., y FELISAVERDEJO, M. (2000). "Analysing student interaction processes in order to improve collaboration. The DEGREE approach" en International Journal of Artificial Intelligence in Education, vol. $11, n^{\circ} 3$, pp. 221-241.

HENKE, N. "The age of analytics: Competing in a data-driven world| McKinsey," [En línea]. Disponible: https://www.mckinsey.com/business-functions/mckinsey-analytics/our-insights/the-age-of-analyticscompeting-in-a-data-driven-world. [Último acceso: 11 Jun 2021].

KEIS, O., HELBIG, H., STREB, J., y HILLE, K. (2014). "Influence of blue-enriched classroom lighting on students ' cognitive performance" en Trends in Neuroscience and Education, vol. 3, no 3-4, pp. 8692.

KWALLEK, N., LEWIS, C. M., LIN-HSIAO, J. W. D., y WOODSON, H. (1996). "Effects of nine monochromatic office interior colors on clerical tasks and worker mood" en Color Research \& Application, vol. 21, n 6, pp.448-458.

LAMA M., SÁNCHEZ E. (2007). "Introducción a la monografía Técnicas de IA aplicadas a la Educación" en I. Artificial, Inteligencia artificial: revista iberoamericana de inteligencia artificial, vol. 11, $\mathrm{n}^{\circ} 33$.

MAHNKE, F. H. (1996). Color, environment, and human response: an interdisciplinary understanding of color and its use as a beneficial element in the design of the architectural environment. New York: Van Nostrad Reinhold.

PAN, Y. (2016). "Heading toward Artificial Intelligence 2.0" en Engineering, vol. 2, n 4, pp. 409-413. 
La Inteligencia Artificial y su contribución a la optimización de la atención y memoria de los estudiantes a través del diseño del aula- Artificial Intelligence and its contribution to optimising students' attention and memory through classroom design.

REINSEL, D., GANTZ J., y RYDNING J. (2018). "The Digitization of the World From Edge to Core".

ROLL, I., y WYLIE, R. (2016) "Evolution and Revolution in Artificial Intelligence in Education" en International Journal of Artificial Intelligence in Education, vol. 26, $\mathrm{n}^{\circ}$ 2, pp. 582-599.

RUS, V., D'MELLO, S., HU X., y GRAESSER, A. (2013). "Recent Advances in Conversational Intelligent Tutoring Systems" en Al Magazine, vol. 34, n 3, p. 42.

SEIDMAN, L. J., BREITER, H. C., GOODMAN, J. M., GOLDSTEIN, J. M., WOODRUFF, P. W., O'CRAVEN, K., ... ROSEN, B. R. (1998). "A functional magnetic resonance imaging study of auditory vigilance with low and high information processing demand" en Neuropsychology, vol. 12, $n^{\circ} 4, p$. 505-518.

SHEARER C. (2000). "El modelo CRISP-DM: el nuevo plan para la minería de datos, almacenamiento de los datos" en Journal of Data Warehousing, vol.5, $n^{\circ} 4$, pp. 13-22.

SMOLDERS, K. C., DE KORT, Y. A., y CLUITMANS, P. J. M. (2012). "A higher illuminance induces alertness even during office hours: findings on subjective measures, task performance and heart rate measures", en Physiology \& Behavior, vol. 107, $\mathrm{n}^{\circ}$ 1, pp. 7-16.

STOKES, B. (2005). "Videogames have changed: time to consider 'Serious Games'?", en The Development Education Journal, vol. 11, n 3, p. 12.

STONE, N. J. (2003). "Environmental view and color for a simulated telemarketing task", en Journal of Environmental Psychology, vol. 23, issue 1, pp. 63-78.

TARTAGLIA, A., y TRESSO, E. (2002). "An automatic evaluation system for technical education at the University level" en IEEE Transactions on Education, vol. 45, n 3, pp. 268-275.

TIMMINS, M.J. (2016). "Letting Artificial Intelligence in Education Out of the Box: Educational Cobots and Smart Classrooms" en International Journal of Artificial Intelligence in Education, vol. 26, $\mathrm{n}^{\circ} 2$, pp. 701-712.

VANLEHN, K. (2011). "The Relative Effectiveness of Human Tutoring, Intelligent Tutoring Systems, and Other Tutoring Systems" en Educational Psychology, vol. 46, nº 4, pp. 197-221.

VANNINI, N. (2011). "FearNot!': a computer-based anti-bullying-programme designed to foster peer intervention" en European Journal of Psychology of Education, vol. 26, $\mathrm{n}^{\circ}$ 1, pp. 21-44.

VIOLA, A. U., JAMES, L. M., SCHLANGEN, L. J., y DIJK, D. J. (2008). "Blue-enriched white light in the workplace improves self-reported alertness, performance and sleep quality" en Scandinavian Journal of Work, Environment \& Health, vol. 34, n 4, p. 297-306.

XIA, T., SONG, L., WANG, T. T., TAN, L., y MO, L. (2016). "Exploring the effect of red and blue on cognitive task performances" en Frontiers in Psychology, vol. 7, 784. 